\title{
Regards sur l'action publique en Afrique
}

\author{
Introduction
}

Philippe Lavigne Delville

\section{OpenEdition \\ Journals}

Édition électronique

URL : http://journals.openedition.org/anthropodev/540

DOI : 10.4000/anthropodev.540

ISSN : 2553-1719

\section{Éditeur}

Presses universitaires de Louvain

\section{Édition imprimée}

Date de publication : 1 mai 2017

Pagination : 13-22

ISBN : 979-10-93476-04-9

ISSN : 2276-2019

\section{Référence électronique}

Philippe Lavigne Delville, « Regards sur l'action publique en Afrique», Anthropologie \& développement [En ligne], 45 | 2017, mis en ligne le 01 novembre 2017, consulté le 10 décembre 2020. URL : http:// journals.openedition.org/anthropodev/540; DOI : https://doi.org/10.4000/anthropodev.540

\section{cc) (†)}

La revue Anthropologie \& développement est mise à disposition selon les termes de la Licence Creative Commons Attribution 4.0 International. 


\title{
Regards sur l'action publique en Afrique
}

\author{
Introduction
}

\author{
Philippe Lavigne Delville
}

Que signifie parler d'action publique en Afrique, et plus largement dans les pays "sous régime d'aide " où l'aide internationale, ses institutions, ses financements, jouent un rôle structurel ? Un regard en termes d'action publique apporte-t-il des éclairages nouveaux sur les politiques et les interventions de développement, étudiées de longue date par la socioanthropologie du développement ?

Intitulé " la fabrique de l'action publique dans les pays 'sous régime d'aide' ", le $12^{\mathrm{e}}$ colloque international de I'APAD $^{1}$ avait pour objectif de débattre de ces questions, à la fois en rassemblant des travaux récents sur les questionnements classiques de la socio-anthropologie du développement (les interventions de développement à l'échelle locale, les services publics et collectifs, etc.) et en explorant des objets encore nouveaux, comme la façon même dont sont produites et négociées les politiques d'aide et les politiques publiques nationales. Il visait aussi à décloisonner les recherches sur ces objets, entre socio-anthropologie du développement, sociologie politique de l'action publique et science politique.

Les textes réunis dans ce dossier entament la valorisation scientifique du colloque et explorent la problématique de l'action publique en Afrique.

\footnotetext{
${ }^{1}$ Ce colloque s'est déroulé à Cotonou, du 19 au 21 novembre 2015, en partenariat avec le LADYD (Laboratoire d'analyse des dynamiques de développement, Faculté des sciences agronomiques, université d'Abomey-Calavi), le LASDEL (Laboratoire d'études et de recherche sur les dynamiques sociales et le développement local), l'université d'Abomey Calavi et l'IRD (Institut de recherche pour le développement).
} 
Politiques de développement, projets de développement : une action publique internationalisée et extravertie

La socio-anthropologie du développement a principalement étudié les projets de développement, et ne s'intéresse que depuis quelques années aux politiques publiques en tant que telles.

Le projet de développement est, depuis l'époque coloniale, le mode privilégié d'intervention publique. L'intervention coloniale est une intervention en archipel, qui se concentre sur les espaces jugés utiles (les centres urbains, les zones aménagées pour l'irrigation, les zones de cultures de rente) et où la " mise en valeur » est confiée à des sociétés d'aménagement ou de développement publiques ou parapubliques dans ce qui est déjà « une administration à deux vitesses »(Blundo, 2011).

Lancée à l'époque du New Deal aux États-Unis, la Tennessee Valley Authority est l'archétype (très empirique au début; cf. Hargrove, 1994) des projets d'aménagement régional intégré (Garel, 2003). Ce sera le modèle de projets coloniaux de " mise en valeur ». Les États indépendants multiplient ces projets, confiés à des sociétés régionales de développement, avant qu'ils soient disqualifiés pour leur inefficacité et leur coût pour la puissance publique, à la veille des ajustements structurels, et quasiment abandonnés par la suite. James Scott (1998) qualifiera de "haut modernisme " ce mélange de volonté techniciste de rationalisation des sociétés et de la nature, et de pouvoirs autoritaires.

Le projet de développement était ainsi à l'origine un instrument des politiques étatiques, confié à des institutions étatiques. La figure du projet telle qu'elle s'est développée dans les années 1990 à 2000 s'inscrit dans le contexte spécifique de l'après ajustement, où l'État était marginalisé et où, dans les politiques des bailleurs de fonds, des projets sortis de l'administration se substituent à des politiques nationales (Naudet, 1999). C'est à cette phase que correspond la multiplication - parfois désordonnée - de projets de toutes sortes, la généralisation des financements de court terme et du principe de mise en concurrence, la systématisation des outils de management de projet, et finalement la diffusion vers les collectivités locales, les organisations paysannes, les ONG nationales, de cette culture bureaucratique du projet. 
Les projets de développement comme substituts aux politiques nationales sont eux-mêmes mis en cause à partir des années 2000 : les effets pervers de cette multiplication de projets deviennent ingérables; cherchant à se relégitimer après la " fatigue de l'aide " qui a suivi la chute du mur de Berlin, les institutions d'aide mettent à nouveau en avant les États et affirment agir en appui aux politiques. L'appropriation, l'ownership (Raffinot, 2010), deviennent des maîtres-mots. La Déclaration de Paris entérine ce changement de doctrine, qui ne se traduit que partiellement par des changements de pratique (Wood, Betts, Etta et al., 2011).

Désormais, par un nouveau mouvement de balancier, l'aide programme, les financements groupés (" basket funding " en appui aux politiques sectorielles) voire l'aide budgétaire, sectorielle ou non (Arnal, 2007), sont valorisés. Les bailleurs de fonds tentent de se coordonner autour de programmes pluriannuels, couplant objectifs stratégiques et institutionnels, et objectifs quantitatifs en termes de résultats. Ils réduisent leurs financements aux ONG pour la mise en œuvre de leurs propres projets de terrain, et les orientent vers une fonction de plaidoyer ou d'expérimentation d'une part, et de mise en œuvre des politiques en sous-traitance au sein des programmes nationaux d'autre part.

Le retour des politiques s'effectue cependant sous de fortes contraintes. Elles doivent être approuvées par les bailleurs de fonds et respecter les cadrages macro-économiques, dans ce que Bergamaschi (2008) appelle " $a$ donor-driven ownership ». Les Objectifs du millénaire pour le développement et la Déclaration de Paris entérinent une " coresponsabilité " des États et des bailleurs de fonds aboutissant à un "increasing entanglement of donor institutions and recipient administrative systems » (Whitfield et Fraser, 2009 : 19).

Une part importante de la définition des politiques publiques ou de leur mise en œuvre relève désormais d'acteurs nationaux fortement internationalisés et multipositionnés (Diallo, 2012). Les ministères sectoriels retrouvent un plus grand contrôle sur les politiques, mais les mécanismes de financement continuent largement à suivre des procédures spécifiques, quand les tentatives de coupler procédures nationales et procédures des bailleurs de fonds n'aboutissent pas à une "co-production du retard » (Blundo, 2015). Bien plus, la volonté de suivre les procédures financières nationales amène les bailleurs de fonds à 
pousser à une restructuration des modalités même de la gestion publique, accentuant leur ingérence au cœur du fonctionnement des États (Raffinot et Samuel, 2006).

Dans ce modèle, le projet ne disparaît pas, il reste une modalité privilégiée de mise en œuvre des politiques, au sein de programmes d'envergure nationale. La managérialisation n'est pas remise en cause, bien au contraire. Au sein même de cette gouvernance internationalisée, les configurations varient. À côté de ces programmes (programme décennal de l'éducation, etc.), certains secteurs relevant de problèmes globaux comme les grandes endémies sont traités par des dispositifs spécifiques, largement transnationaux, comme le Fonds mondial contre le sida, le paludisme et la tuberculose (Eboko, 2015).

Depuis le début des années 2000, les pays africains sont dans une situation de coproduction assumée de l'action publique entre États et bailleurs de fonds (et parfois ONG internationales), entérinant et institutionnalisant la souveraineté limitée des États dans des formes ambiguës de gouvernance internationalisée. Les configurations varient cependant (Fraser et Whitfield, 2009). La gestion de la dépendance à l'aide relève, pour Bayart, de stratégies d'extraversion, qui sont historiquement un mode d'articulation des élites africaines au reste du monde (Bayart, 1999). La capacité des bailleurs de fonds à imposer leurs conceptions est débattue. Les bailleurs de fonds sont eux-mêmes prisonniers d'un jeu où ils ont besoin de dépenser pour exister, et où les temporalités ne sont pas en leur faveur. De plus, focaliser le regard sur l'aide amène à laisser dans l'ombre les stratégies politiques qui se jouent à travers, et parfois sous, ces processus de négociation des politiques. L'analyse de la production des politiques foncières en Ouganda (Gay, 2016) montre ainsi comment le pouvoir central doit négocier avec les élites politiques régionales et le parlement, dans des processus complexes, très politiques, où l'aide, ses cadrages, ses experts, sont finalement bien peu présents.

Sur différents champs, on observe des tentatives de la part des institutions étatiques pour garder ou reprendre le contrôle de ressources stratégiques, pour remettre en cause des politiques de décentralisation ou de transfert de pouvoir et de ressources qui avaient été mises en place 
sous la forte impulsion de l'aide au plus fort de la faiblesse des États ${ }^{2}$. Par ailleurs, l'action de l'État ne se limite pas aux secteurs liés à l'aide. En coordination, en substitution ou en opposition aux actions des institutions étatiques, d'autres acteurs, nationaux ou internationaux, prennent en charge la définition et le traitement de problèmes considérés comme publics ou collectifs. Bref, prendre acte du fait que l'action publique est pluri-acteurs, internationalisée et extravertie oblige à s'interroger de façon empirique sur les configurations politiques et institutionnelles, sur la structure des arènes, sur les acteurs en jeu et leurs relations, sur les controverses et leurs enjeux et à explorer ces modalités ambiguës de coproduction de l'action publique en pays 'sous régime d'aide'.

Politiques publiques, action publique, gouvernabilité : présentation du dossier

Partout dans le monde, le tournant néolibéral a déplacé les rapports entre État, secteur privé et secteur associatif dans la production et la mise en œuvre des politiques publiques, à partir de modèles très différents, liés aux trajectoires politiques des États. La contribution du secteur privé et du secteur associatif est davantage valorisée. Les logiques managériales se développent. Ces recompositions prennent des formes pour partie spécifiques dans les pays africains, du fait de leur histoire politique et institutionnelle, et du fait de la prégnance de l'aide internationale.

Les quatre contributions réunies dans ce dossier explorent différentes facettes de la question de l'action publique en Afrique. Partant du questionnement global du colloque, Philippe Lavigne Delville plaide pour une socio-anthropologie de l'action publique dans les pays 'sous régime d'aide'. Pour lui, une telle perspective conforte et élargit à la fois les questionnements de la socio-anthropologie du développement. Ce que I'on appelle "politiques ou actions de développement", dit-il, relève largement de ce qui est ailleurs qualifié de politiques publiques, de secteur d'économie sociale et solidaire. Déplacer le questionnement permet un regard nouveau sur cette action publique largement extravertie, en

${ }^{2}$ Cf. sur la faune sauvage en Tanzanie : Benjaminsen, Goldman, Minwary et al., 2013. 
distinguant mieux dans la mise en évidence des gaps et des contradictions, ce qui relève des contradictions structurelles de l'action publique de ce qui relève des effets de configurations spécifiques aux pays sous régime d'aide. Multiplications d'acteurs, luttes interinstitutionnelles, problèmes d'ingouvernabilité liés à l'explosion des acteurs de l'action publique, sont en effet des processus globaux. Poser les questions en termes d'action publique amène de plus à s'intéresser davantage à l'amont des politiques et des projets, à la façon dont les cadrages internationaux sont produits, aux processus internationalisés de fabrique des politiques publiques où enjeux de policy (politique sectorielle), de politics (de lutte pour le pouvoir), de polity (de contrat social) s'imbriquent étroitement. Ce qui apporte un regard nouveau tant sur ces formes de gouvernance internationalisée que sur le politique en Afrique. Une telle perspective, soutient Lavigne Delville, nécessite un dialogue critique avec la sociologie politique de l'action publique, qui a développé des concepts et des outils d'analyse pertinents, même s'ils ne peuvent évidemment pas être importés sans une mise à l'épreuve critique.

Donner à voir ces questionnements et ces approches à un public apadien est l'objectif du deuxième article de ce dossier. À partir de ses travaux et de la conférence inaugurale qu'il a donnée lors du colloque, Philippe Zittoun présente une approche "pragmatique" de l'action publique, qui s'intéresse aux processus par lesquels des énoncés de problèmes sont formulés et propagés, et par lesquels des acteurs porteurs d'enjeux tentent d'assembler des problèmes et des solutions, de rendre traitables des problèmes. II montre l'importance du travail de cadrage et d'argumentation, inhérent aux politiques publiques, et l'importance à accorder aux pratiques discursives, aux discours en acte. II montre à quel point ce processus, même lorsqu'il emprunte un discours technicisé, est profondément politique. Philippe Zittoun resitue cette approche dans un panorama des travaux en science politique et sociologie politique sur l'action publique, montrant l'éclatement de ce champ en de multiples courants, illustrant la spécificité des recherches de "policy process analysis ", qui cherchent non pas à proposer des solutions aux décideurs mais à élucider les processus eux-mêmes, et soulignant les concepts clés mis en avant au cours de l'histoire de la discipline. Bien au-delà de la défense et de l'illustration d'une façon d'aborder la question de l'action 
publique, ce texte est aussi une introduction à ce champ de recherche, dont il montre la diversité et le foisonnement.

Portant sur des cas béninois, les deux articles suivants illustrent deux facettes des questionnements en termes d'action publique. Apportant sa contribution aux débats sur la coproduction de l'action publique entre acteurs hétérogènes (Olivier de Sardan, Abdelkader, Diarra et al., 2011), Issifou Abou Moumouni s'interroge sur les collaborations qui se sont institutionnalisées entre forces de gendarmerie et associations de chasseurs dans le nord-est du Bénin. Au cours des années 1990, les chasseurs, confrérie traditionnelle des sociétés rurales d'Afrique des savanes, se sont organisés en associations de lutte contre la délinquance. Ils sont régulièrement mobilisés par les forces de l'ordre dans la lutte contre le banditisme, et en particulier les coupeurs de route. Leur connaissance de la brousse, leur capacité à affronter le danger, en font des auxiliaires précieux, et eux-mêmes se considèrent comme tels. On observe ainsi depuis une vingtaine d'années une certaine coproduction de la sécurité mobilisant dispositifs étatiques et acteurs non étatiques, ces derniers complétant l'action des forces de l'ordre dans des contextes ou sur des registres où celles-ci ne peuvent pas agir. La nature semi-officieuse de leur intervention permet aussi aux membres des associations de chasseurs de s'affranchir des contraintes auxquelles sont soumises les forces de l'ordre, et les chasseurs sont réputés responsables d'exécutions extrajudiciaires de coupeurs de route, maquillées en légitime défense lors des attaques. Les autorités étatiques valorisent la contribution des chasseurs à la sécurité publique, les enjoignent de remettre les suspects à la gendarmerie, tout en couvrant les pratiques illégales qui suppléent leurs propres limites. Loin de traduire un affaiblissement de l'État, ces formes de coproduction sont, pour Issifou Abou Moumouni, une stratégie de construction locale de l'État.

Héloïse Valette, Fabrice Gangneron et Alain Bonnassieux s'intéressent quant à eux à la fabrique d'une politique sectorielle, celle de l'eau potable en milieu rural et en milieu périurbain au Bénin. Dans ce secteur, la Conférence de Dublin a, en 1994, entériné le principe marchand : le service de l'eau doit s'autofinancer par la vente de l'eau. Ce principe est transcrit dans la politique nationale béninoise quelques années après, au tournant des années 2000 , et remet en cause le cadre institutionnel antérieur de la 
"gestion communautaire". Mais les doctrines internationales ne se traduisent pas mécaniquement (Delpeuch, 2009). À chaque passage d'une échelle à l'autre, les principes sont traduits et reformulés, les enjeux varient, les acteurs aussi. La réforme du secteur de l'eau potable au Bénin est proche de celle qu'ont connue, à peu près à la même époque, les pays voisins : responsabilité communale du service, délégation de sa mise en œuvre à des organisations privées. Mais les cadres du ministère de l'Eau ont imposé une vision restrictive des acteurs privés, valorisant les entrepreneurs privés et rejetant la petite ouverture légale sur la délégation de gestion à des associations d'usagers. Ils se sont aussi battus pour conserver une centralisation des appels d'offre pour la réalisation des nouvelles adductions d'eau et ainsi conserver un contrôle sur les moyens financiers, réduisant en pratique les prérogatives communales. À l'échelle locale, la réorganisation institutionnelle impose que les communes récupèrent la propriété des équipements existants, au détriment des organisations villageoises qui en assuraient la gestion. Pour garantir le paiement de la redevance qui leur est due, elles limitent la durée des contrats de délégation, mettent en péril la rentabilité pour les entrepreneurs. On observe ainsi des recompositions partielles du service d'eau potable, très variables d'une commune à l'autre, sans que la délégation aux entrepreneurs résolve vraiment les problèmes de qualité du service qui avaient justifié la critique de la gestion communautaire.

Transferts de modèles par les bailleurs de fonds et réinterprétations nationales, enjeux politiques des réformes en termes de rapports État/collectivités locales et de pouvoir des administrations sectorielles, coproduction de l'action publique avec des acteurs non étatiques: ces deux textes illustrent des facettes importantes des politiques publiques et de l'action publique en Afrique, et des rapports entre institutions étatiques et institutions non étatiques, nationales et internationales. Ils posent la question de la nature de cette gouvernance pluri-acteurs peu régulée et des problèmes de gouvernabilité qu'elle engendre. D'autres dimensions seront explorées dans un prochain dossier: la gouvernance locale des services et les concurrences et complémentarités entre acteurs à l'échelle locale, les mobilisations sociales et la mise à l'ordre du jour des réformes, les jeux d'instrumentalisations réciproques entre État et bailleurs de fonds, la publicisation de problèmes par des acteurs hors de l'aide, etc. 


\section{Bibliographie}

ARNAL J.-F., 2007, « Comment la mise en oeuvre de la Déclaration de Paris a servi de tremplin à la réforme du processus d'aide budgétaire globale au Ghana ", Afrique contemporaine, 223-224(3-4) : 325-339.

BAYART J.-F., 1999, "L'Afrique dans le monde: une histoire d'extraversion ", Critique internationale, 5(1) : 97-120.

BENJAMINSEN T.A., GOLDMAN M.J., MINWARY M.Y. et al., 2013, "Wildlife Management in Tanzania: State Control, Rent Seeking and Community Resistance ", Development and Change, 44(5) : 1087-1109.

BERGAMASCHI I., 2008, Mali : Patterns and Limits of Donor-driven Ownership, Global Economic Governance Program Working Papers $n^{\circ} 41$, Oxford, University College, $39 \mathrm{p}$.

BLUNDO G., 2011, « Une administration à deux vitesses. Projets de développement et construction de l'État au Sahel ", Cahiers d'études africaines, 202203(2-3) : 427-452.

BLUNDO G., 2015, La coproduction du retard. Anthropologie de l'aide budgétaire au Cameroun et au Niger, Paris, Agence française de développement, $45 \mathrm{p}$.

DELPEUCH T., 2009, "Comprendre la circulation internationale des solutions d'action publique: panorama des policy transfer studies », Critique internationale, 43(2) : 153-165.

DIALLO R.N., 2012, "Élites administratives, aide internationale et fabrique de l'action publique de la conservation au Mozambique ", Politique africaine, 126(2) : 143-161.

EBOKO F., 2015, Vers une matrice de l'action publique en Afrique ? Approche transsectorielle de l'action publique en Afrique contemporaine, Questions de Recherche / Research Question n 45, CERI, 40 p.

FRASER A. et WHITFIELD L., 2009, « Understanding Contemporary Aid Relationship ", in Whitfield L. (éd.), The Politics of Aid, Oxford, Oxford University Press : 74-107.

GAREL G., 2003, Le management de projet, Coll. Repères, Paris, La Découverte.

GAY L., 2016, À la recherche de l'hégémonie: la fabrique très politique des politiques publiques foncières en Ouganda sous le régime du National 
Resistance Movement (NRM), entre changement et inertie, Doctorat en science politique, université de Montpellier, Montpellier, $764 \mathrm{p}$.

HARGROVE E.C., 1994, Prisoners of myth: The leadership of the Tennessee Valley Authority, 1933-1990, Princeton, Princeton University Press.

NAUDET D., 1999, Trouver des problèmes aux solutions. Vingt ans d'aide au Sahel, Paris, OCDE.

OLIVIER DE SARDAN J.-P., ABDELKADER A., DIARRA A. et al., 2011, Gouvernance locale. La délivrance de quatre biens publics dans trois communes nigériennes, Études et Travaux $n^{\circ}$ 95, Niamey, LASDEL, $39 \mathrm{p}$.

RAFFINOT M., 2010, "L'appropriation (ownership) des politiques de développement: de la théorie à la pratique ", Mondes en développement, 149(1) : 87-104.

RAFFINOT M. et SAMUEL B., 2006, « Les cadres de dépenses à moyen terme : un instrument utile pour les pays à faible revenu ? ", Statéco, $100: 105-120$.

SCOTT J., 1998, Seeing like a State. How Certain Schemes to Improve the Human Condition Have Failed, New Haven \& London Yale University Press.

WHITFIELD L. et FRASER A., 2009, "Introduction: Aid and Sovereignty ", in Whitfield L. (éd.), The Politics of Aid, Oxford, Oxford University Press : 126.

WOOD B., BETTS J., ETTA F. et al, 2011, L'évaluation de la Déclaration de Paris, phase 2, rapport final, Copenhague, Institut danois d'études internationales, $251 \mathrm{p}$.

\title{
MNNW
}

\author{
Philippe Lavigne Delville est socio-anthropologue \\ directeur de recherche à l'IRD et président de l'APAD \\ E-mail : philippe.lavignedelville@ird.f
}

